Check for updates

Cite this: J. Mater. Chem. B, 2021, 9, 7472

Received 6th May 2021,

Accepted 9th August 2021

DOI: $10.1039 / \mathrm{d} 1 \mathrm{tb} 01020 \mathrm{~d}$

rsc.li/materials-b

\title{
Monitoring the formation of a colloidal lipid gel at the nanoscale: vesicle aggregation driven by a temperature-induced mechanism $\dagger$
}

\author{
Kirian Talló, (D) * Ramon Pons, (D) César González and Olga López
}

\begin{abstract}
Colloidal gels made of lipid vesicles at highly diluted conditions have been recently described. The structure and composition of this type of material could be especially relevant for studies that combine model lipid membranes with proteins, peptides, or enzymes to replicate biological conditions. Details about the nanoscale events that occur during the formation of such gels would motivate their future application. Thus, in this work we investigate the gelation mechanism, which consists of a lipid dispersion of vesicles going through a process that involves freezing and heating. The appropriate combination of techniques (transmission electron microscopy, differential scanning calorimetry and synchrotron small angle X-ray scattering) allowed in-depth analysis of the different events that give rise to the formation of the gel. Results showed how freezing damaged the lipid dispersion, causing a polydisperse suspension of membrane fragments and vesicles upon melting. Heating above the lipids' main phase transition temperature promoted the formation of elongated tubular structures. After cooling, these lipid tubes broke down into vesicles that formed branched aggregates across the aqueous phase, obtaining a material with gel characteristics. These mechanistic insights may also allow finding new ways to interact with lipid vesicles to form structured materials. Future works might complement the presented results with molecular dynamics or nuclear magnetic resonance experiments.
\end{abstract}

\section{Introduction}

Aggregation and fusion of lipid vesicles are among the most fundamental processes in biological organisms. ${ }^{1}$ Studies have shown that agents like proteins, ions, and polymers can allow vesicles to interact in proximity and even merge. ${ }^{2-5}$ Traditionally, these membrane-based events have been studied in model systems from a fundamental perspective using lipid vesicles to replicate biological structures like cell membranes. ${ }^{6-9}$ Systems with other morphologies, like bicelles, ${ }^{10}$ have also been used to study membrane fusion and aggregation, providing additional properties to the system, such as the ability to interact with magnetic fields and incorporate proteins. ${ }^{11-14}$

Knowledge of biological processes has led to the development of materials able to mimic biological conditions, which provide a better compatibility and specificity with the interactions occurring in living organisms. ${ }^{15-18}$ Nonetheless, research towards structured materials based on the aggregation of lipid

Department of Surfactants and Nanobiotechnology, Institute of Advanced Chemistry of Catalonia (IQAC-CSIC), C/Jordi Girona 18-26, 08034 Barcelona, Spain.

E-mail:kirian.tallo@cid.csic.es

$\dagger$ Electronic supplementary information (ESI) available. See DOI: 10.1039/d1tb01020d vesicles has barely been explored. Some works have reported the formation of structured aggregates composed of phospholipid vesicles, leading to a percolated state or fractal organisation. ${ }^{19,20}$

Reports about the formation dynamics of structured suspensions mainly focus on polymer or clay particles rather than lipid vesicles. ${ }^{21,22}$ Numerical and experimental data of these works discuss how electrostatic balance between particles affects the formation and structure of colloidal gels and glasses. In a previous study, we reported the possibility of forming a colloidal gel through the aggregation of lipid vesicles composed of phosphatidylcholine and oleic acid. ${ }^{23}$ Such remarkable behaviour takes place under dilute conditions and without requiring gelling agents. Our last work reported the effect of charge in their microscopic structure and rheological behaviour of these types of systems by forming colloidal lipid gels composed of phosphatidylcholine and 1,2-dioleoyl-3-trimethylammoniumpropane (DOTAP). ${ }^{24}$ Results indicated that the formation and integrity of the gel are related to the attractive-repulsive interactions between those particles.

The supramolecular organization of colloidal lipid gels may open the possibility to use these materials as mimetic membrane models for works that require self-assembled lipid 
structures, such as those that study membrane proteins, peptides or enzymes. ${ }^{25-28}$ For the case of peptides, the interaction of their positive charge with negatively charged lipids could be relevant to study events of permeabilization and rupture of the gel lipid membranes. In addition, knowledge about the mechanisms that govern the formation of colloidal lipid gels would motivate future research on similar types of lipid structured materials with potential biomedical applications.

Thus, in the present study we investigate the distinct stages involved in the formation mechanism of colloidal lipid gels. For this purpose, we have characterised a dispersion of positively charged vesicles during different moments of their gelation through transmission electron microscopy (TEM), differential scanning calorimetry (DSC) and synchrotron X-ray scattering. Such complete characterisation of the procedure at the nanoscale has been relevant to understand the crucial events related to the formation of these gels. The next steps might require NMR and molecular dynamics experiments to complement the mechanistic description or utilize techniques like confocal microscopy to explore the interactions occurring above the nanoscale.

\section{Materials and methods}

\subsection{Materials}

Hydrogenated soy phosphatidylcholine (HSPC; Phospholipon ${ }^{\circledR}$ 90H) and 1,2-dioleoyl-3-trimethylammonium-propane (DOTAP) were acquired from Lipoid GmbH (Ludwigshafen, Germany). Phospholipon ${ }^{\circledR} 90 \mathrm{H}$ is a combination of 1,2-distearoyl-snglycero-3-phosphocholine (DSPC) (ca. 85\%) and 1-palmitoyl-2stearoyl-3-phosphocholine (PSPC) (ca. 15\%). Chloroform (Grade: ACS, ISO, Reag. Ph Eur) was purchased from Merck (Darmstadt, Germany).

\subsection{Preparation of the gel}

The assays performed in this work started from an aqueous dispersion of lipid vesicles composed of HSPC and DOTAP prepared with the thin film hydration method, ${ }^{29}$ following ultrasound bath (J.P. Selecta Ultrasons H-D, $330 \mathrm{~W}, 50 \mathrm{~Hz}$ ) at room temperature for $15 \mathrm{~min}$. Sample volume for sonication was $5 \mathrm{~mL}$. The total lipid concentration was $5 \% \mathrm{w} / \mathrm{w}$ with a molar ratio HSPC/DOTAP set to $20: 1$. Gel preparation follows a five-step process starting from the initial dispersion of lipid vesicles (a) leading to three intermediates (b, c, and d) and the colloidal lipid gel (e) at the end:

Step 1. Preparation of a dispersion of lipid vesicles: (a)

Step 2. Freezing of (a) from 25 to $-20{ }^{\circ} \mathrm{C}$ : (b)

Step 3. Melting of (b) from $-20{ }^{\circ} \mathrm{C}$ to $25{ }^{\circ} \mathrm{C}$ : (c)

Step 4. Heating of (c) from 25 to $70{ }^{\circ} \mathrm{C}$ : (d)

Step 5. Cooling of (d) from $70{ }^{\circ} \mathrm{C}$ to $25{ }^{\circ} \mathrm{C}$ : (e)

While the formation of (a) involved agitation and sonication, the following steps only required thermal energy. For DSC and $\mathrm{X}$-ray scattering, the temperature was decreased $3{ }^{\circ} \mathrm{C} \mathrm{min}^{-1}$ from 25 to $-20{ }^{\circ} \mathrm{C}$ (step 2), maintained $30 \mathrm{~min}$ at $-20{ }^{\circ} \mathrm{C}$, increased $5{ }^{\circ} \mathrm{C} \min ^{-1}$ from -20 to $70{ }^{\circ} \mathrm{C}$ (steps 3 and 4), maintained $10 \mathrm{~min}$

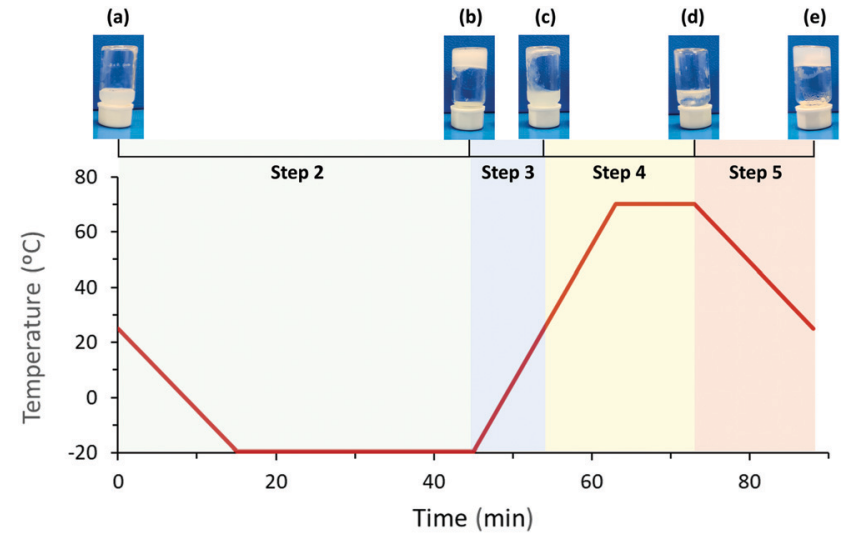

Fig. 1 Diagram representing the variation of temperature applied to the sample with time. for illustrative purposes, a caption of the initial (a) intermediate (b, c and d) and final (e) appearance of the sample is shown at different time points.

at $70{ }^{\circ} \mathrm{C}$, and decreased $3{ }^{\circ} \mathrm{C} \min ^{-1}$ from 70 to $25{ }^{\circ} \mathrm{C}$ (step 5). Fig. 1 displays a schematic representation of the temperature variation with time and the macroscopic aspect of the sample after each step of the procedure.

\subsection{Differential scanning calorimetry (DSC)}

DSC measurements were performed by triplicate using an $821 \mathrm{E}$ Mettler Toledo (Greifensee, Switzerland) calorimeter. Aliquots of approximately $5 \mu \mathrm{L}$ from the lipid dispersion (a) and a control sample of miliQ water were placed inside $40 \mu \mathrm{L}$ aluminium DSC pans sealed hermetically. The DSC curves were analysed by the STAR 9.2 Software (Mettler Toledo). Melting enthalpies of sample and control were calculated from the integration of endothermic bands and were normalised by the amount of water.

\subsection{Small angle X-ray scattering (SAXS)}

$\mathrm{X}$-ray scattering measurements were carried out with synchrotron radiation at the BL11-NCD-SWEET beamline at ALBA (Cerdanyola del Vallès, Barcelona, Spain). The lipid dispersion (a) was loaded into a glass capillary of $1.5 \mathrm{~mm}$ and placed into a Linkam capillary stage used to control the temperature during steps 2 to 5 of the gelation procedure. The temperature was decreased $3{ }^{\circ} \mathrm{C} \min ^{-1}$ from 25 to $-20{ }^{\circ} \mathrm{C}$ (step 2), maintained $30 \mathrm{~min}$ at $-20{ }^{\circ} \mathrm{C}$, increased $5{ }^{\circ} \mathrm{C} \mathrm{min}^{-1}$ for heating scans from -20 to $70{ }^{\circ} \mathrm{C}$ (steps 3 and 4), maintained $10 \mathrm{~min}$ at $70{ }^{\circ} \mathrm{C}$, and decreased $3{ }^{\circ} \mathrm{C} \mathrm{min}^{-1}$ from 70 to $25{ }^{\circ} \mathrm{C}$ (step 5).

Samples were aligned to the X-ray beam with an energy of $12.4 \mathrm{keV}$. Small-angle patterns were acquired using a Pilatus $1 \mathrm{M}$ detector placed at $2.71 \mathrm{~m}$ from the sample. The sample was exposed 5 seconds for each time frame. Silver behenate and chromium trioxide were used as standards to calibrate the small angle and wide angle detectors, respectively. The initial data treatment was performed using the software provided by the beamline. Background subtraction was performed using the spectra of water-filled capillary and empty capillary at 25 and $70{ }^{\circ} \mathrm{C}$; small correction factors were applied to avoid 
negative values. For the frozen samples, the same liquid background was subtracted but with significant correction factors.

The subtracted small angle X-ray scattering SAXS spectra were fitted to scattering curves based on Gaussian electronic profiles as before, including the contribution of lamellar stacks based on Caillé description. ${ }^{30,31}$ In a previous work ${ }^{24}$ we used a model of asymmetric bilayers to give account of the observed SAXS spectra, however, in the present case the asymmetric bilayer model did not provide significantly improved fitting. This bilayer profile was complemented by using the method of separated form factors for polydisperse vesicles as described by Pencer et al. ${ }^{32}$ As we lack a model for separate form factors for bilayers arranged in tubular form, we have calculated few spectra by Fourier transformation of the pair distribution distance function and compared the results with those of spherical vesicles with the same bilayer characteristics. More details of those calculations are given in the ESI. $\dagger$ As will be shown later, significant differences only appear at very small $q$ vectors.

\subsection{Methylcellulose staining and TEM visualisation}

Aliquots of $0.4 \mathrm{~mL}$ of lipid samples (a), (c), (d) and (e), corresponding to initial, intermediates, and final states, were prepared beforehand in closed vials employing similar conditions to DSC and X-ray scattering experiments. Step 2 of the protocol was performed by storing the vials at $-20{ }^{\circ} \mathrm{C}$ during $1 \mathrm{~h}$. For step 3, samples were placed inside a warm bath at $25{ }^{\circ} \mathrm{C}$ for $15 \mathrm{~min}$. Afterwards, the samples were heated to $70{ }^{\circ} \mathrm{C}$ during $20 \mathrm{~min}$ for step 4. Finally, gels were left cooling at $25{ }^{\circ} \mathrm{C}$ for $20 \mathrm{~min}$.

Each sample was deposited for 1 minute over formvarcoated 200 mesh nickel grids that were previously activated with UV light for $5 \mathrm{~min}$. Sample excess was removed with filter paper, and grids were deposited over a droplet of the staining solution made with a combination of $0.4 \%$ uranyl acetate and $2 \%$ methylcellulose cooled over ice for $10 \mathrm{~min}$. Note that sample (d) required a previous fixation step at $70{ }^{\circ} \mathrm{C}$ before staining with methylcellulose. For this purpose, the grid with the sample was deposited for 1 min over a droplet of $4 \%$ uranyl acetate at $70{ }^{\circ} \mathrm{C}$, and excess was removed with filter paper. When all samples were stained, excess solution was removed using filter paper, and grids were air-dried at room temperature. The combination of methylcellulose with a heavy metal (uranyl) was based on previous works performed by James Naismith and John Lucocq research groups. ${ }^{33,34}$ Samples were observed using a TEM JEOL J1010 $80 \mathrm{kV}$ at the Electron Cryomicroscopy Unit of the University of Barcelona (CCiTUB, Barcelona, Spain).

\section{Results and discussion}

\subsection{Differential scanning calorimetry (DSC)}

DSC measures heat flow associated with transitions in systems as a function of temperature and time, like freezing/melting events or transitions associated to the rearrangement of lipid bilayers. In this experiment, we used DSC to assess the relative

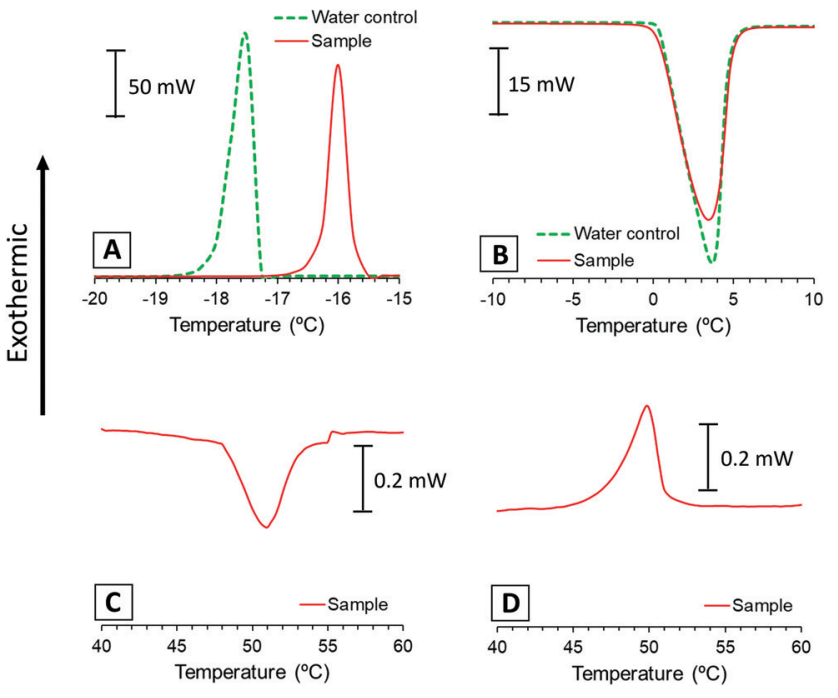

Fig. 2 DSC scan of the lipid dispersion sample and the water control: exothermic bands associated with freezing of step 2 (A), endothermic bands associated with melting of step 3 (B), and the endothermic band corresponding to the main phase transition of the lipid membranes from $L_{\beta}$ to $L_{\alpha}$ in step 4 (C) and from $L_{\alpha}$ to $L_{\beta}$ in step 5 (D).

amount of water that freezes during step 2 of the gelation procedure and the main phase transition temperature $\left(T_{\mathrm{m}}\right)$ of the lipid membranes. For this purpose, we ran a calorimetry scan of the lipid dispersion sample (a) following steps 2 to 4 of the procedure, and we compared the calorimetric transitions of the sample with an ultrapure water control.

Fig. 2A presents the exothermic bands corresponding to the water freezing process of sample and control. These bands show discrepant values on their freezing points $\left(-16{ }^{\circ} \mathrm{C}\right.$ and $-17.5^{\circ} \mathrm{C}$ ) because the crystallisation of water is likely affected by supercooling phenomena. On the other hand, endothermic bands corresponding to the ice melting process of the sample and the control appear over the same temperatures (Fig. 2B). Thus, we integrated melting bands for enthalpy comparison because these are more reliable, while freezing might be affected by other phenomena.

Table 1 shows the normalised melting enthalpies calculated from the area under the endothermic bands of Fig. 2B and the corresponding replicates (Fig. S1B, ESI $\dagger$ ). These values correspond to the heat absorbed during the melting process and correlate with the relative amount of frozen water. The similarity between normalised enthalpy values of lipid sample and pure water control indicates that the whole amount of

Table 1 Melting enthalpies normalised per amount of water, which can be correlated to the amount of previously frozen water. Values are the means and standard deviation (SD) of three replicates $(n=3)$

\begin{tabular}{llr}
\hline & Normalised enthalpy $\left(\mathrm{J} \mathrm{g}^{-1}\right)$ & \\
\cline { 2 - 3 } & Mean & SD \\
\hline Water control & -341 & 2 \\
Sample & -341 & 1
\end{tabular}


water froze in the lipid dispersion. These results were used to assess the lamellarity of the vesicles present in the dispersion, as previously reported by Kaasgaard and co-workers. ${ }^{35}$ Their experiments showed that suspensions of unilamellar vesicles froze almost entirely in the first freeze-thawing cycle. In contrast, multilamellar vesicles presented incomplete water freezing at first, which increased with multiple freezethawing cycles.

Overall, when the temperature is decreased below $0{ }^{\circ} \mathrm{C}$, bulk water freezes faster than intravesicular due to the likeliness of presenting impurities that act as heterogeneous nucleation agents. $^{36}$ Then, the superconcentrated frozen bulk phase causes an osmotic gradient between intravesicular and bulk regions that induces vesicle dehydration and eventual rupture of their membrane due to water efflux. ${ }^{37,38}$ In unilamellar vesicles, this results in complete freezing, while multilamellar systems require successive freeze-thawing events.

As described previously, the similar melting enthalpy values between sample and control (Table 1) indicate complete water freezing in the lipid dispersion. Thus, we hypothesise that only unilamellar vesicles are present in the dispersion right before freezing occurs, which then might follow dehydration and damage to their membranes.

The calorimetric scan was continued up to $70{ }^{\circ} \mathrm{C}$ to identify the main phase transition temperature of the lipid phase (Fig. 2C). This plot shows an endothermic band around $51{ }^{\circ} \mathrm{C}$ that can be associated with the main phase transition of the lipid mixture of HSPC/DOTAP. For comparison purposes, a thermogram of the initial dispersion (a) was performed from 25 to $70{ }^{\circ} \mathrm{C}$, showing the same transition also centred at $51{ }^{\circ} \mathrm{C}$ (Fig. S2, ESI $\dagger$ ). These bands usually appear in bilayered systems when transitioning from the solid-like phase $\mathrm{L}_{\beta}$ to the liquid crystalline phase $\mathrm{L}_{\alpha}$. Although bilayers made only of HSPC have a reported transition around $54{ }^{\circ} \mathrm{C},{ }^{29}$ the presence of DOTAP disrupts the HSPC packing and diminishes the transition temperature. ${ }^{39,40}$ During the cooling of the sample (Fig. 2D), an exothermic band appears around $51{ }^{\circ} \mathrm{C}$ corresponding to the same main phase transition of the lipid membranes but on the opposite direction (from $\mathrm{L}_{\alpha}$ to $\mathrm{L}_{\beta}$ phase). In addition to the main band, systems composed purely of HSPC present a pretransition $\left(\mathrm{L}_{\beta^{\prime}}\right)$ around $46{ }^{\circ} \mathrm{C} .{ }^{29}$ However, we hypothesize that the lack of pretransition in the present results could be caused by the interference of DOTAP in the membrane. The alteration of the transition temperature and packing of the membranes makes more evident that the formation of the gel is strongly dependent on the lipids used. The influence of lipids was already present in a previous study, where were determined how the amount of a charged lipid affected the rheology and microstructure this type of system. ${ }^{24}$

\subsection{Transmission electron microscopy (TEM)}

TEM was used to evaluate morphology, size and aggregation of the different structures formed in the lipid systems (a), (c), (d) and (e) that are present during the gelation procedure.

Fig. 3A and B show the initial dispersion of lipid vesicles at $25{ }^{\circ} \mathrm{C}(\mathrm{a})$. These images present unilamellar vesicles whose size
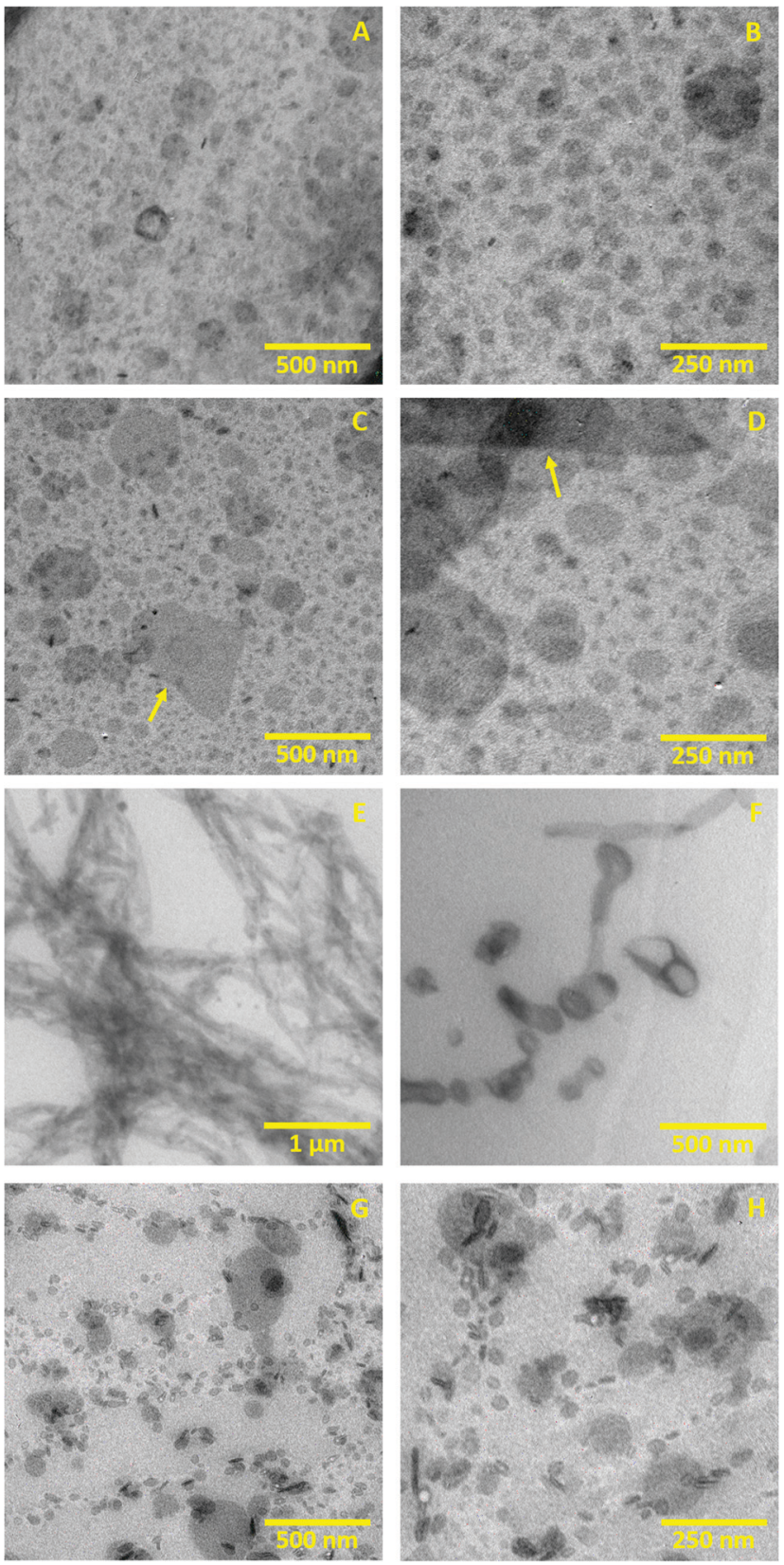

Fig. 3 TEM images of the lipid dispersion during the different stages of the gelation procedure: initial lipid dispersion (a) (A and B), dispersion after melting at $25^{\circ} \mathrm{C}$ (c) $(\mathrm{C}$ and $\mathrm{D})$, dispersion after heating at $70{ }^{\circ} \mathrm{C}$ (d) (E and $\left.\mathrm{F}\right)$ and colloidal gel (e) $(\mathrm{G}$ and $\mathrm{H})$.

ranges from $50 \mathrm{~nm}$ to $150 \mathrm{~nm}$ in diameter. The unilamellar morphology of these vesicles is probably caused by the electrostatic charge of DOTAP: The positive charge at the vesicle surface creates repulsion between membranes, making unfavourable a multilamellar aggregation. ${ }^{41}$ These micrographs agree with DSC results which also indicate unilamellarity of the vesicles until solution freezes.

Fig. 3C and D illustrate the sample after melting at $25{ }^{\circ} \mathrm{C}(\mathrm{c})$. These images present particles with higher size variability than system (a), showing vesicles and small lipid fragments that range from 20 to $200 \mathrm{~nm}$ in size. These micrographs also reveal 
prominent structures with different morphology that extend up to $700 \mathrm{~nm}$ (yellow arrows). The size variability and the small membrane fragments probably result from the freezing procedure that caused the dehydration and rupture of vesicles (as discussed in the DSC Section). Upon ice melting, these shattered vesicles and the membrane fragments can rehydrate again to form aggregates of distinct size, giving rise to a polydisperse system. Similar results have also been reported with dispersions of multilamellar liposomes, where freezethawing is employed to break the lipid membranes, reducing the lamellarity and particle size. ${ }^{42}$

Fig. 3E, corresponding to the intermediate system (d), shows how the lipid vesicles acquired a tubular morphology after heating the sample to $70{ }^{\circ} \mathrm{C}$. The tubes that form those entangled aggregates have a diameter that goes from 40 to $150 \mathrm{~nm}$. With higher magnification, we identified few vesicle aggregates lining up in a tube-like shape, as shown in Fig. 3F. We hypothesise that after increasing the temperature above $T_{\mathrm{m}}$, the lipid vesicles and fragments that resulted from freezing can fuse, forming tubular structures. Despite the electrostatic charge stabilises the system, the defects in the membranes caused by freezing and the thermal energy could allow adhesion and fusion. ${ }^{6,43,44}$ The formation of bilayered tubes from adhesion of phospholipid vesicles has been reported on different occasions employing divalent ions, cationic nanoparticles or electric fields, among other strategies. ${ }^{45-47}$

Fig. $3 \mathrm{G}$ and $\mathrm{H}$ show the nanostructure of the final gel after cooling the dispersion from $70{ }^{\circ} \mathrm{C}$ to $25{ }^{\circ} \mathrm{C}$, corresponding to the system (e). These images present small vesicles from 30 to $150 \mathrm{~nm}$ diameter that, as opposed to the initial dispersion (Fig. 3A and B), are not homogeneously distributed in space. Instead, they form branched aggregates that resemble a fractallike structure and probably cause the gel-like rheology. This heterogeneous organisation could result from the segmentation of the tubular structures caused by temperature decrease: when the temperature drops below $T_{\mathrm{m}}$, the membranes return to the rigid $\mathrm{L}_{\beta}$ phase, and the bilayers are more likely to organise again into spherical vesicles to regain stability. Although segmentation of vesicular tubes is not a typical process, some works have reported a similar phenomenon known as pearling. In this event, external agents induce the contraction of tubular vesicles at periodic regions, ${ }^{48,49}$ which can divide into vesicles in specific cases. ${ }^{50}$

Overall, the gelation procedure starts with a fluid homogeneous dispersion of vesicles and ends with a structured gel where vesicles remain aggregated in a heterogeneous manner. In previous works, we determined that the presence of charged lipids is crucial to form this type of gels. ${ }^{23,24}$ In the present system, vesicles experience attractive van der Waals forces and repulsive electrostatic interactions caused by the positively charged DOTAP. When a repulsive-attractive balance is present, gelation is more likely to occur through the formation of particle clusters of finite size. ${ }^{21}$ However, the suspension of vesicles (a) does not show signs of gelation over time despite the charge balance. Instead, it has been evidenced that the formation of the gel requires freezing (dehydrating and breaking vesicles) and heating above $T_{\mathrm{m}}$ (fusing vesicles into tubular aggregates). We hypothesize that once the system is cooled down, tubular structures break, forming vesicles in close distance that can remain aggregated in a stable network, likely due to the electrostatic balance. Such mechanism, where the lipid membranes get disrupted in order to achieve gelation, open the possibility of a potential use of these gels as model systems in works that involve the fragmentation of lipid membranes, like those that employ antimicrobial or amyloid peptides. $^{51,52}$

\subsection{Small angle X-ray scattering (SAXS)}

In these experiments, we used synchrotron X-ray radiation to monitor the events during the gelation procedure of the lipid dispersion. The temperature was varied with time inside the capillary that contained the sample, while X-ray scattering data were acquired at different steps of the process. Fig. 4 plots the experimental SAXS results, the fitting models, and the electron density profiles of the corresponding lipid bilayers. Note that in this figure, there are two spectra acquired at $-20{ }^{\circ} \mathrm{C}$, one before sample freezing (red circles) and the other after the sample was completely frozen (green triangles). The different parameters corresponding to the models fitted are presented in Table 2.

At the starting of the gel process, the sample at $24{ }^{\circ} \mathrm{C}$ presents a SAXS spectrum with a main wide band followed by two more minor reflections characteristic of uncorrelated unilamellar vesicles (Fig. 4A) ${ }^{23,41}$ The leftmost side of the spectra $\left(q<0.5 \mathrm{~nm}^{-1}\right)$ presents a bend that can be fitted using a model of polydisperse vesicles with mean radius $\left(R_{\mathrm{v}}\right)$ of $50.5 \mathrm{~nm}$ that agrees with TEM results presented in Fig. 3A and $3 \mathrm{~B}$. The subsequent cooling of the sample down to $-20{ }^{\circ} \mathrm{C}$ (before freezing) results in a spectrum barley unchanged, indicating the same type of system with a similar vesicle size $\left(R_{\mathrm{v}}=48.5 \mathrm{~nm}\right)$. The electron density profiles of both spectra (Fig. 4B) present a dip at the centre of the bilayer corresponding to the methyl terminal groups. This sharp dip indicates that hydrocarbon tails of the lipids forming the bilayers are not interdigitated. Considering that the sample is still below the $T_{\mathrm{m}}\left(51^{\circ} \mathrm{C}\right)$, we believe that this lack of interdigitation corresponds to membranes organised in a solidlike phase $\left(\mathrm{L}_{\beta}\right) .^{53}$

Fig. 4A also shows the spectrum of the frozen sample at $-20{ }^{\circ} \mathrm{C}$ and the spectrum while being heated at $-7{ }^{\circ} \mathrm{C}$, below the melting point. The four equidistant reflections of both spectra (where Bragg $d$-spacing is slightly bigger at $-7{ }^{\circ} \mathrm{C}$ ) correspond to bilayers organised in a multilamellar structure. ${ }^{30}$ According to the fit, membranes at $-20{ }^{\circ} \mathrm{C}$ and $-7{ }^{\circ} \mathrm{C}$ form stacks of 12 correlated bilayers (and a domain size of about $75 \mathrm{~nm}$ ). These profiles are adequately represented, although the fit presents lower goodness (high $\chi^{2}$ ), probably due to an insufficient resolution of the model. Overall, these lamellar profiles support the idea explored in the microscopy section that lipid vesicles dehydrate upon freezing due to osmotic pressure and collapse into multilamellar stacks of membranes (see the values of associated water in Table 2). Aside from the prominent lamellar bands, these spectra also show additional peaks at $q=2.24 \mathrm{~nm}^{-1}\left(-20{ }^{\circ} \mathrm{C}\right.$ and $\left.-7{ }^{\circ} \mathrm{C}\right)$ and 

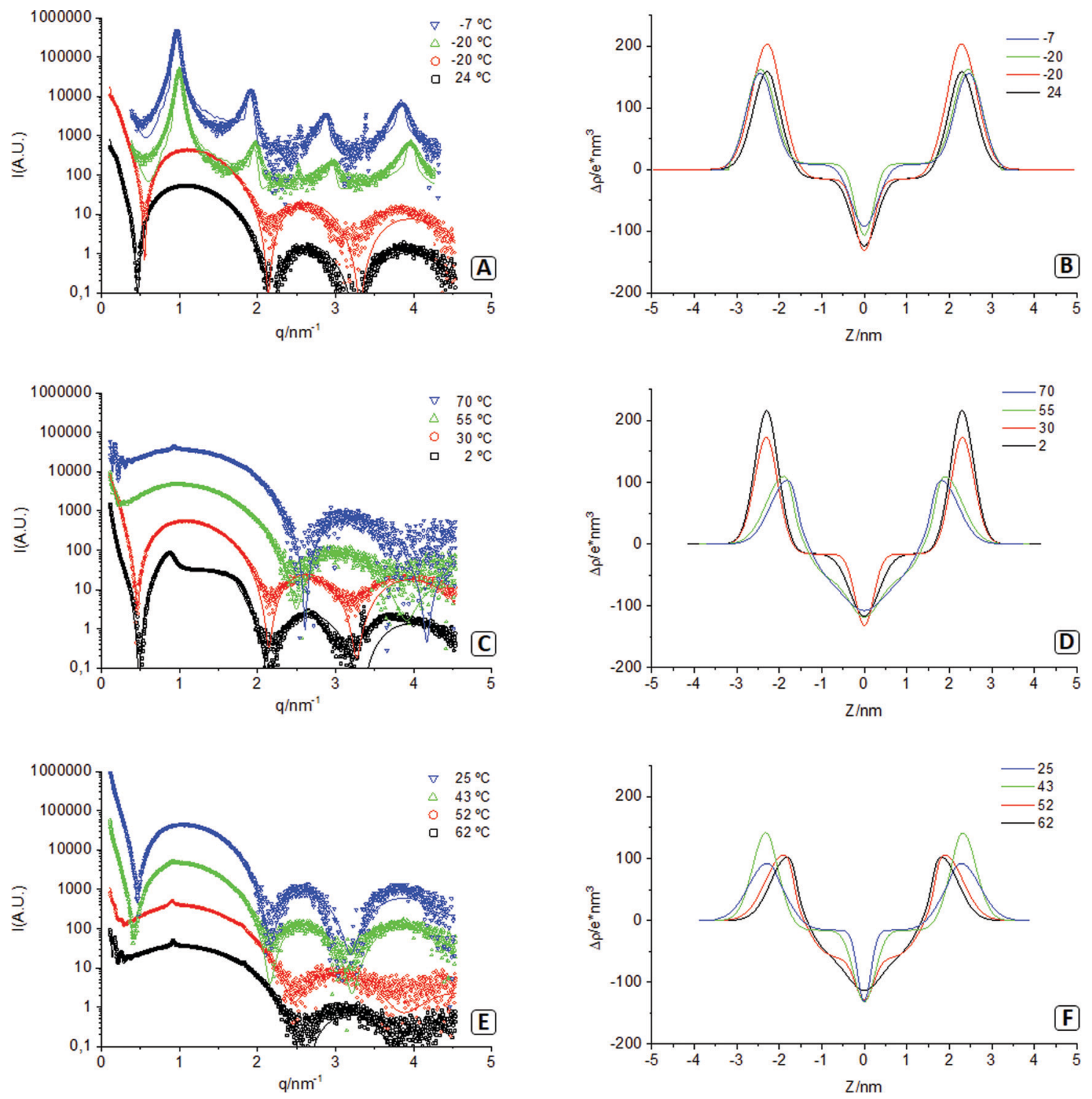

Fig. 4 Left: experimental SAXS profiles of the sample at various stages of the gelation procedure (markers) and the corresponding fitting (solid lines). Right: electronic density profiles of the corresponding bilayers.

$q=3.1 \mathrm{~nm}^{-1}\left(-7^{\circ} \mathrm{C}\right)$. The nature of those peaks is unknown, although they could correspond to an additional degree of order in the polar headgroup plane. The electron density profiles (Fig. 4B) of both frozen samples show that lipid membranes remain in a non-interdigitated manner. Note in this figure that frozen water has a lower electron density than methylene chains $\left(\rho_{\mathrm{w}}<\rho_{\mathrm{CH}_{2}}\right)$, but liquid water has a higher value $\left(\rho_{\mathrm{w}}>\rho_{\mathrm{CH}_{2}}\right)$ this produces significant changes in the form factor due to sign change of the contrast.

Fig. 4C shows the spectra that appear when the sample continues being heated up to $70{ }^{\circ} \mathrm{C}$. At $2{ }^{\circ} \mathrm{C}$, the ice starts melting, and the correlation between bilayers is severely reduced; at $30{ }^{\circ} \mathrm{C}$, the peaks have disappeared, and the correlation between bilayers is lost. This indicates that the lamellar stacks of membranes that were formed upon freezing have now rehydrated again into vesicular structures resulting in a profile $\left(30{ }^{\circ} \mathrm{C}\right)$ very similar to that of the sample at the start of the procedure $\left(24{ }^{\circ} \mathrm{C}\right)$. It only differs in the small $q$ region, where the sample at $30{ }^{\circ} \mathrm{C}$ no longer needs the use of a separate form factor for the vesicles, likely due to the very high polydispersity, agreeing with TEM (Fig. 3C and D). Further heating at $55{ }^{\circ} \mathrm{C}$ (above $T_{\mathrm{m}}$ ) shows a different trend, where the minimum at $0.5 \mathrm{~nm}^{-1}$ has disappeared, and the minima at 2.2 and $3.2 \mathrm{~nm}^{-1}$ have shifted to 2.5 and $3.8 \mathrm{~nm}^{-1}$. This different profile reflects the transition of the hydrophobic chains from a solid-like phase $\left(\mathrm{L}_{\beta}\right)$ to a liquid crystalline phase $\left(\mathrm{L}_{\alpha}\right)$ that causes interdigitation of their hydrophobic chains, as seen in the electron density profile (Fig. 4D). ${ }^{30}$ This is also accompanied by a significant increase in area per molecule and hydration water (see Table 2).

Further increase of temperature to $70{ }^{\circ} \mathrm{C}$ results in two additional features. First, a tiny but sharp peak appearing at 
Table 2 Parameters corresponding to the SAXS fitting models at different temperatures $\left({ }^{\circ} \mathrm{C}\right)$ following the experimental order. Please note that the first $-20{ }^{\circ} \mathrm{C}$ corresponds to the unfrozen sample

\begin{tabular}{|c|c|c|c|c|c|c|c|c|c|c|c|c|}
\hline & 24 & -20 & -20 & -7 & 2 & 30 & 55 & 70 & 62 & 52 & 43 & 25 \\
\hline$\overline{\chi^{2}}$ & 8.7 & 14.4 & 47 & 45.1 & 9.5 & 6.2 & 3.9 & 2.6 & 3.9 & 1.28 & 1.1 & 1.4 \\
\hline$d$ & & & 6.34 & 6.54 & 7.55 & & & 6.76 & 6.85 & 6.93 & 7.03 & \\
\hline$\eta$ & & & 0.012 & 0.013 & 0.141 & & & 0.62 & 0.56 & 0.64 & 0.04 & \\
\hline$N_{\mathrm{c}}$ & & & 12 & 12 & 4 & & & 36 & 25 & 16.3 & 4.5 & \\
\hline$N_{\mathrm{u}}$ & & & 0 & & 5.9 & & & 610 & 216 & 93 & 108 & \\
\hline$\sigma_{\mathrm{h}}$ & 0.33 & 0.37 & 0.32 & 0.31 & 0.28 & 0.28 & 0.42 & 0.41 & 0.39 & 0.44 & 0.32 & 0.44 \\
\hline$\rho_{\mathrm{h}}$ & 489 & 534 & 467 & 461 & 549 & 505 & 438 & 430 & 434 & 434 & 473 & 423 \\
\hline$Z_{\mathrm{h}}$ & 2.28 & 2.28 & 2.42 & 2.46 & 2.29 & 2.30 & 1.88 & 1.80 & 1.81 & 1.88 & 2.31 & 2.28 \\
\hline$\sigma_{\mathrm{c}}$ & 0.28 & 0.22 & 0.21 & 0.31 & 0.34 & 0.22 & 0.34 & 0.46 & 0.40 & 0.21 & 0.25 & 0.16 \\
\hline$A_{\mathrm{m}}$ & 0.43 & 0.43 & 0.39 & 0.40 & 0.42 & 0.42 & 0.63 & 0.67 & 0.67 & 0.64 & 0.42 & 0.43 \\
\hline$N_{\mathrm{w}}$ & & & 1 & 3 & 13 & & & 30 & 31 & 29 & 9 & \\
\hline$\rho_{\mathrm{w}}$ & 332 & 329 & 307 & 307 & 334 & 332 & 329 & 327 & 329 & 329 & 332 & 332 \\
\hline$\rho_{\mathrm{CH}_{2}}$ & 316 & 316 & 316 & 316 & 316 & 316 & 292 & 288 & 290 & 292 & 316 & 316 \\
\hline$\rho_{\mathrm{CH}_{3}}$ & 225 & 225 & 225 & 225 & 225 & 225 & 220 & 220 & 220 & 220 & 225 & 225 \\
\hline$R_{\mathrm{V}}$ & 50.5 & 48.5 & & & & & 28.5 & 44.0 & 44.9 & 45.4 & 40.5 & 35.5 \\
\hline PI & 0.14 & 0.13 & & & & & 0.15 & 0.076 & 0.076 & 0.090 & 0.20 & 0.22 \\
\hline
\end{tabular}

$\chi^{2}$ reduced chi-squared, $d$ Bragg distance $(\mathrm{nm}), \eta$ Caillé parameter, $N_{\mathrm{c}}$ number of correlated lamellae, $N_{\mathrm{u}}$ number of uncorrelated lamellae, $\sigma_{\mathrm{h}}$ amplitude $(\mathrm{nm})$ of the Gaussian corresponding to the polar head with electronic density $\rho_{\mathrm{h}}\left(\mathrm{e} \mathrm{nm}^{-3}\right), Z_{\mathrm{h}}$ position of the polar heads (nm) with respect to the centre of the bilayer, $\sigma_{\mathrm{c}}$ amplitude of the Gaussian representing the terminal methyl groups, $A_{\mathrm{m}}$ area per lipid molecule (nm ${ }^{2}$ ), $N_{\mathrm{w}}$ number of water molecules associated to the polar head, $\rho_{\mathrm{w}}, \rho_{\mathrm{CH} 2}, \rho_{\mathrm{CH} 3}$ electronic density $\left(\mathrm{e} \mathrm{nm}^{-3}\right)$ corresponding to the water, methylene and terminal methyl groups as used in the fits, $R_{\mathrm{v}}$ radii or the vesicles $(\mathrm{nm})$ and polydispersity index PI.

$q=1.0 \mathrm{~nm}^{-1}$ that corresponds to a small percentage of the lipid phase organised in correlated bilayers $(<2 \%)$. The second feature corresponds to an oscillation appearing in the small $q$ region (below $0.4 \mathrm{~nm}^{-1}$ ). The origin of this oscillation cannot be univocally associated with a particular feature. We have explored several options, among them, the long-distance correlation of flat lamellae and the form factor of spherical vesicles with a low polydispersity. Although all fitted models are based on vesicles, the TEM images illustrate the formation of tubular structures at $70{ }^{\circ} \mathrm{C}$. Due to the limited experimental SAXS window, the differences between vesicles and tubes are hardly discernible.

To confirm the formation of tubular structures by SAXS, we have developed a model of bilayered cylinders, which was compared with a model of spherical vesicles built using equivalent parameters (see the ESI $\dagger$ for more details on the models and the findings). These models were fitted to the small $\mathrm{q}$ region of the experimental SAXS data from the sample at $70{ }^{\circ} \mathrm{C}$ (Fig. 5). Note that the superimposed oscillations in this figure, centred at $q=0.8,1.6$, 3.2 , and $4.8 \mathrm{~nm}^{-1}$ arise from the reduced number of sizes used to simulate polydispersity. From these fittings, it can be concluded that the period of the oscillations using a tubular model fits better the experimental data than using an equivalent model of spherical vesicles. This reinforces the theory that the fragmented vesicles from the freeze-thawing process can fuse into tubular structures upon heating above $T_{\mathrm{m}}$. It must be noted that the oscillation at small q could also correspond to the correlation of flat lamellae with Bragg distances of about $77 \mathrm{~nm}$. However, such conformations were not observed by electron microscopy in order to support this hypothesis.

The spectra acquired during the cooling of the sample down to $25{ }^{\circ} \mathrm{C}$ are presented in Fig. $4 \mathrm{E}$. At $62{ }^{\circ} \mathrm{C}$ and $52{ }^{\circ} \mathrm{C}$, patterns are very similar, but when the temperature is decreased to $43{ }^{\circ} \mathrm{C}$ (below $T_{\mathrm{m}}$ ), the minima at $0.5 \mathrm{~nm}^{-1}$ is recovered, and the other

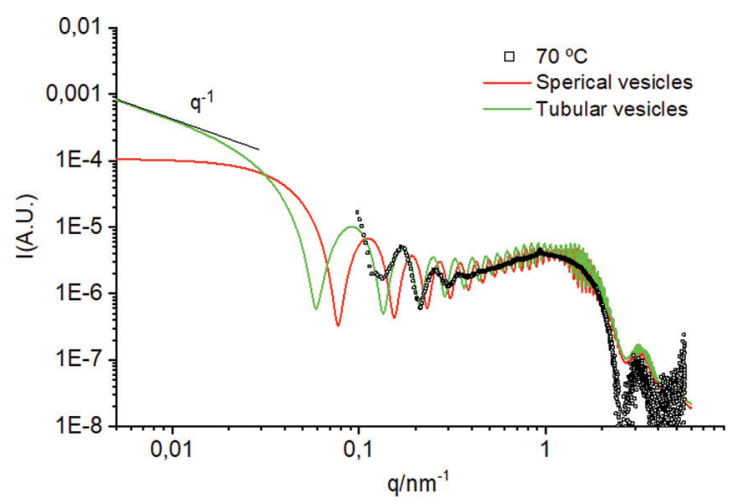

Fig. 5 Comparison between a model of spherical vesicles and tubular vesicles fitted on the experimental SAXS profile of the sample at $70^{\circ} \mathrm{C}$.

two minima are displaced to the original positions again. Besides, electron density profiles (Fig. $4 \mathrm{~F}$ ) show how membranes lose the interdigitation as they transition back to a solidlike phase $\left(\mathrm{L}_{\beta}\right)$. At $43{ }^{\circ} \mathrm{C}$, a certain degree of multilamellarity is still retained (corresponding to the peak at $q=1.0 \mathrm{~nm}^{-1}$ ), but the oscillation at small $q$ has been completely lost. When the gel is formed at the end of the process $\left(25^{\circ} \mathrm{C}\right)$, the multilamellarity is lost, and the electron density profile and the rest of the parameters approximate to those from the start of the procedure $\left(24{ }^{\circ} \mathrm{C}\right)$. This similarity is congruent with TEM images, where the structural unit is the lipid vesicle, regardless of their branched/gel-like organisation.

\section{Conclusions}

This work explored the relevance of each step involved in the gelation mechanism of a suspension of lipid vesicles. 
In previous works, we reported that a proper electrostatic balance between structures was necessary to form these types of gels; with the present data, we evidence that the preparation protocol is equally relevant. These findings suggest a combined effect between freezing and heating steps of the procedure that turn the lipid dispersion into a colloidal gel. Freezing results in the dehydration and rupture of the vesicles and heating above the $T_{\mathrm{m}}$ induces fusion of the damaged membranes into elongated tubular structures. The eventual formation of the gel results in branched aggregates of vesicles that correlate with the characteristic viscoelastic behaviour of the material. Thus, the particularity on this gelation method lies in fundamental membrane-based events that occur during freezing and heating, differing from other colloidal gelation mechanisms that typically involve polymeric particles.

With the presented results, we aim to inspire the use of the gel in related areas such as the study of peptide or enzymatic interactions in mimetic model membranes. For this purpose, these works should support the mechanistic insights here discussed with NMR or molecular dynamic studies. In addition, we expect that the description of the mechanism may allow finding approaches to control the interaction of lipid vesicles, leading to novel biocompatible materials. Despite our results focusing on the interactions occurring at the nanoscale, those next works could address the mechanistic details occurring at the microscale with techniques like confocal microscopy.

\section{Author contributions}

Kirian Talló: writing - original draft, conceptualization, investigation. Ramon Pons: software, formal analysis, funding acquisition. César González: software, formal analysis. Olga López: funding acquisition, supervision, writing - review \& editing.

\section{Conflicts of interest}

There are no conflicts to declare.

\section{Acknowledgements}

The authors wish to thank Sonia Pérez-Rentero from IQAC for DSC measurements and Yolanda Muela from CCITUB for TEM sample preparation. The authors are also grateful to Lipoid $\mathrm{GmbH}$ for kindly providing the lipid components used in this work.

This research was funded by the Ministerio de Ciencia e Innovación, Spain, grants CTQ2017-88948-P, RTC-2016-4957-1 and Fondo Europeo de Desarrollo Regional (FEDER).

SAXS experiments were performed at NCD beamline at ALBA Synchrotron with the collaboration of ALBA staff.

\section{References}

1 E. H. Chen and E. N. Olson, Unveiling the mechanisms of cell-cell fusion, Science, 2005, 308, 369-373.
2 T. Weber, B. V. Zemelman, J. A. McNew, B. Westermann, M. Gmachl, F. Parlati, T. H. Söllner and J. E. Rothman, SNAREpins: minimal machinery for membrane fusion, Cell, 1998, 92, 759-772.

3 F. Bordi and C. Cametti, Salt-induced aggregation in cationic liposome aqueous suspensions resulting in multi-step self-assembling complexes, Colloids Surf., B, 2002, 26, 341-350.

4 T. Tanaka and M. Yamazaki, Membrane Fusion of Giant Unilamellar Vesicles of Neutral Phospholipid Membranes Induced by La3+, Langmuir, 2004, 20, 5160-5164.

5 N. de Lange, F. A. M. Leermakers and J. M. Kleijn, Selflimiting aggregation of phospholipid vesicles, Soft Matter, 2020, 16, 2379-2389.

6 S. Nir, J. Bentz, J. Wilschut and N. Duzgunes, Aggregation and fusion of phospholipid vesicles, Prog. Surf. Sci., 1983, 13, 1-124.

$7 \mathrm{~S}$. Ohki and H. Ohshima, Interaction and aggregation of lipid vesicles (DLVO theory versus modified DLVO theory), Colloids Surf., B, 1999, 14, 27-45.

8 D. J. Estes, S. R. Lopez, A. O. Fuller and M. Mayer, Triggering and Visualizing the Aggregation and Fusion of Lipid Membranes in Microfluidic Chambers, Biophys. J., 2006, 91, 233-243.

9 K. Komorowski, A. Salditt, Y. Xu, H. Yavuz, M. Brennich, R. Jahn and T. Salditt, Vesicle Adhesion and Fusion Studied by Small-Angle X-Ray Scattering, Biophys. J., 2018, 114, 1908-1920.

10 C. R. Sanders and R. S. Prosser, Bicelles: a model membrane system for all seasons?, Structure, 1998, 6, 1227-1234.

11 M. Bortolus, M. De Zotti, F. Formaggio and A. L. Maniero, Alamethicin in bicelles: Orientation, aggregation, and bilayer modification as a function of peptide concentration, Biochim. Biophys. Acta, Biomembr., 2013, 1828, 2620-2627.

12 G. Lai, K. M. Forti and R. Renthal, Kinetics of lipid mixing between bicelles and nanolipoprotein particles, Biophys. Chem., 2015, 197, 47-52.

13 T. N. Sut, S. Park, Y. Choe and N.-J. Cho, Characterizing the Supported Lipid Membrane Formation from CholesterolRich Bicelles, Langmuir, 2019, 35, 15063-15070.

14 U. H. N. Dürr, M. Gildenberg and A. Ramamoorthy, The Magic of Bicelles Lights Up Membrane Protein Structure, Chem. Rev., 2012, 112, 6054-6074.

$15 \mathrm{X}$. Chen and J. Li, Bioinspired by cell membranes: functional polymeric materials for biomedical applications, Mater. Chem. Front., 2020, 4, 750-774.

16 N. Suresh Kumar, R. Padma Suvarna, K. Chandra Babu Naidu, P. Banerjee, A. Ratnamala and H. Manjunatha, A review on biological and biomimetic materials and their applications, Appl. Phys. A: Mater. Sci. Process., 2020, 126, 445.

17 L. A. Northcutt, A. Suarez-Arnedo and M. Rafat, Emerging Biomimetic Materials for Studying Tumor and Immune Cell Behavior, Ann. Biomed. Eng., 2020, 48, 2064-2077.

18 A. Levin, T. A. Hakala, L. Schnaider, G. J. L. Bernardes, E. Gazit and T. P. J. Knowles, Biomimetic peptide self-assembly 
for functional materials, Nat. Rev. Chem., 2020, 4, 615-634.

19 T. Lahiri, N. S. Kar, A. Chakrabarti and A. K. Dasgupta, Onset of Percolation and Fractal Classification Scheme for Multilamellar Lipid Vesicles, J. Colloid Interface Sci., 1999, 211, 89-95.

20 S. Roldán-Vargas, R. Barnadas-Rodríguez, A. Martín-Molina, M. Quesada-Pérez, J. Estelrich and J. Callejas-Fernández, Growth of lipid vesicle structures: from surface fractals to mass fractals, Phys. Rev. E: Stat., Nonlinear, Soft Matter Phys., 2008, 78, 10902.

21 E. Zaccarelli, Colloidal gels: equilibrium and non-equilibrium routes, J. Phys.: Condens. Matter, 2007, 19, 323101.

22 B. Ruzicka, L. Zulian, E. Zaccarelli, R. Angelini, M. Sztucki, A. Moussaïd and G. Ruocco, Competing Interactions in Arrested States of Colloidal Clays, Phys. Rev. Lett., 2010, 104, 85701.

23 K. Talló, M. Bosch, R. Pons, M. Cocera and O. López, Preparation and characterization of a supramolecular hydrogel made of phospholipids and oleic acid with a high water content, J. Mater. Chem. B, 2020, 8, 161-167.

24 K. Talló, S. Vílchez, R. Pons and O. López, Gels formed from the interaction of lipid vesicles: Influence of charge in their structural and rheological properties, J. Mol. Liq., 2021, 322, 114957.

25 K. Matsuzaki, A. Nakamura, O. Murase, K. Sugishita, N. Fujii and K. Miyajima, Modulation of Magainin 2-Lipid Bilayer Interactions by Peptide Charge, Biochemistry, 1997, 36, 2104-2111.

26 K. Matsuzaki, Why and how are peptide-lipid interactions utilized for self-defense? Magainins and tachyplesins as archetypes, Biochim. Biophys. Acta, Biomembr., 1999, 1462, $1-10$.

27 U. H. N. Dürr, L. Waskell and A. Ramamoorthy, The cytochromes P450 and b5 and their reductases-Promising targets for structural studies by advanced solid-state NMR spectroscopy, Biochim. Biophys. Acta, Biomembr., 2007, 1768, 3235-3259.

28 S. A. Kotler, P. Walsh, J. R. Brender and A. Ramamoorthy, Differences between amyloid- $\beta$ aggregation in solution and on the membrane: insights into elucidation of the mechanistic details of Alzheimer's disease, Chem. Soc. Rev., 2014, 43, 6692-6700.

29 K. Talló, V. Moner, M. De Cabo, M. Cócera and O. López, Vesicular nanostructures composed of oleic acid and phosphatidylcholine: Effect of $\mathrm{pH}$ and molar ratio, Chem. Phys. Lipids, 2018, 213, 96-101.

30 G. Pabst, M. Rappolt, H. Amenitsch and P. Laggner, Structural information from multilamellar liposomes at full hydration: full q-range fitting with high quality x-ray data, Phys. Rev. E: Stat. Phys., Plasmas, Fluids, Relat. Interdiscip. Top., 2000, 62, 4000-4009.

31 E. Haba, A. Pinazo, R. Pons, L. Pérez and A. Manresa, Complex rhamnolipid mixture characterization and its influence on DPPC bilayer organization, Biochim. Biophys. Acta, Biomembr., 2014, 1838, 776-783.
32 J. Pencer, S. Krueger, C. P. Adams and J. Katsaras, Method of separated form factors for polydisperse vesicles, J. Appl. Crystallogr., 2006, 39, 293-303.

33 C. Hacker, J. Asadi, C. Pliotas, S. Ferguson, L. Sherry, P. Marius, J. Tello, D. Jackson, J. Naismith and J. M. Lucocq, Nanoparticle suspensions enclosed in methylcellulose: a new approach for quantifying nanoparticles in transmission electron microscopy, Sci. Rep., 2016, 6, 25275.

34 J. Asadi, S. Ferguson, H. Raja, C. Hacker, P. Marius, R. Ward, C. Pliotas, J. Naismith and J. Lucocq, Enhanced imaging of lipid rich nanoparticles embedded in methylcellulose films for transmission electron microscopy using mixtures of heavy metals, Micron, 2017, 99, 40-48.

35 T. Kaasgaard, O. G. Mouritsen and K. Jørgensen, Freeze/ thaw effects on lipid-bilayer vesicles investigated by differential scanning calorimetry, Biochim. Biophys. Acta, Biomembr., 2003, 1615, 77-83.

36 K. Muldrew and L. E. McGann, The osmotic rupture hypothesis of intracellular freezing injury, Biophys. J., 1994, 66, 532-541.

37 K. Muldrew, J. Schachar, P. Cheng, C. Rempel, S. Liang and R. Wan, The possible influence of osmotic poration on cell membrane water permeability, Cryobiology, 2009, 58, 62-68.

38 K.-I. Izutsu, C. Yomota and T. Kawanishi, Stabilization of Liposomes in Frozen Solutions Through Control of Osmotic Flow and Internal Solution Freezing by Trehalose, J. Pharm. Sci., 2011, 100, 2935-2944.

39 R. B. Campbell, S. V. Balasubramanian and R. M. Straubinger, Phospholipid-cationic lipid interactions: influences on membrane and vesicle properties, Biochim. Biophys. Acta, Biomembr., 2001, 1512, 27-39.

40 H. Matsuki, M. Goto, M. Kusube, N. Tamai and S. Kaneshina, Barotropic Phase Transitions of 1-Palmitoyl2-stearoylphosphatidylcholine Bilayer Membrane, Chem. Lett., 2005, 34, 270-271.

41 N. Kučerka, J. Pencer, J. N. Sachs, J. F. Nagle and J. Katsaras, Curvature effect on the structure of phospholipid bilayers, Langmuir, 2007, 23, 1292-1299.

42 S. Sriwongsitanont and M. Ueno, Effect of Freeze-Thawing Process on the Size and Lamellarity of PEG-Lipid Liposomes, Open Colloid Sci. J., 2010, 4, 1-6.

43 S. Zellmer, G. Cevc and P. Risse, Temperature- and $\mathrm{pH}^{-}$ controlled fusion between complex lipid membranes. Examples with the diacylphosphatidylcholine/fatty acid mixed liposomes, Biochim. Biophys. Acta, Biomembr., 1994, 1196, 101-113.

44 G. Cevc and H. Richardsen, Lipid vesicles and membrane fusion, Adv. Drug Delivery Rev., 1999, 38, 207-232.

45 A. Köth, D. Appelhans, D. Robertson, B. Tiersch and J. Koetz, Use of weakly cationic dendritic glycopolymer for morphological transformation of phospholipid vesicles into tube-like networks, Soft Matter, 2011, 7, 10581-10584.

46 S. Jones, A. Huynh, Y. Gao and Y. Yu, Calcium ion-assisted lipid tubule formation, Mater. Chem. Front., 2018, 2, 603-608.

47 C. Zhu, Y. Zhang, Y. Wang, Q. Li, W. Mu and X. Han, Point-toPlane Nonhomogeneous Electric-Field-Induced Simultaneous 
Formation of Giant Unilamellar Vesicles (GUVs) and Lipid Tubes, Chem. - Eur. J., 2016, 22, 2906-2909.

48 Y. Yu and S. Granick, Pearling of Lipid Vesicles Induced by Nanoparticles, J. Am. Chem. Soc., 2009, 131, 14158-14159.

49 Y.-C. Su and J. Z. Y. Chen, A model of vesicle tubulation and pearling induced by adsorbing particles, Soft Matter, 2015, 11, 4054-4060.

50 T. F. Zhu, K. Adamala, N. Zhang and J. W. Szostak, Photochemically driven redox chemistry induces protocell membrane pearling and division, Proc. Natl. Acad. Sci. U. S. A., 2012, 109, 9828 LP.

51 J. R. Brender, U. H. N. Dürr, D. Heyl, M. B. Budarapu and A. Ramamoorthy, Membrane fragmentation by an amyloidogenic fragment of human Islet Amyloid Polypeptide detected by solid-state NMR spectroscopy of membrane nanotubes, Biochim. Biophys. Acta, Biomembr., 2007, 1768, 2026-2029.

52 D.-K. Lee, J. R. Brender, M. F. M. Sciacca, J. Krishnamoorthy, C. Yu and A. Ramamoorthy, Lipid Composition-Dependent Membrane Fragmentation and Pore-Forming Mechanisms of Membrane Disruption by Pexiganan (MSI-78), Biochemistry, 2013, 52, 3254-3263.

53 S. Leekumjorn and A. K. Sum, Molecular studies of the gel to liquid-crystalline phase transition for fully hydrated DPPC and DPPE bilayers, Biochim. Biophys. Acta, Biomembr., 2007, 1768, 354-365. 